On a resource allocation model

with infinite horizon

\author{
Valeriya Lykina, Sabine Pickenhain \\ and Marcus Wagner \\ Reihe Mathematik \\ M-09/2007 \\ Cottbus University of Technology \\ Institute of Mathematics
}

P. O. B. 1013 44, D-03013 Cottbus, Germany 


\title{
On a Resource Allocation Model with Infinite Horizon
}

\author{
Valeriya Lykina, Sabine Pickenhain and Marcus Wagner \\ Cottbus University of Technology \\ Institute of Mathematics \\ P.O.B. 1013 44, D-03013 Cottbus, Germany \\ lykina@math.tu-cottbus.de,sabine@math.tu-cottbus.de, \\ wagner@math.tu-cottbus.de
}

\begin{abstract}
In this paper we treat a resource allocation model defined on an infinite interval. We show that the solution of the corresponding problem with finite horizon cannot be extended to a solution of the infinite horizon problem, since the resource allocation problem in the unmodified setting does not have a solution on an unbounded interval. To change this situation we bring an additional state constraint into the model which contains a weight function. The new problem, called now the adapted resource allocation problem, has an optimal solution which has been identified by means of the duality concept of Klötzler.
\end{abstract}

Key words: resource allocation, optimal control, infinite horizon, weighted Sobolev and Lebesgue spaces, duality theory.

AMS subject classification: 90 A 80, 46 E 35, 49 J 15, 49 N 15.

\section{Introduction.}

The general interest for infinite horizon optimal control problems has been growing since the seventies of the last century. Many authors dealt with this type of problems, see for instance [1], [2], [4], [8], [14], [16], [18] ff. One reason for this is a plenty of economical, biological and mechanical applications, compare [7], [3], [13], [17] and others. In the present paper we consider the resource allocation problem, given by communication with $\mathrm{H}$. Maurer, formulated on an unbounded interval. This simple model is very demonstrative as for the relation between finite and infinite horizon optimal control problems. The solution of the finite interval problem cannot be extended to a solution 
of the infinite problem, since the last does not even exist. Secondly, none of the admissible trajectories of the infinite horizon problem belongs to the usual Sobolev space what justifies the weighted Sobolev space approach.

Our paper is structured as follows. The second section introduces all necessary notations and definitions. In the section 3 we treat the resource allocation model on a fixed finite time interval and present its solution provided by Pontryagin's maximum principle. The infinite horizon resource allocation problem is considered in the section 4 and it is shown that there is no optimal solution for the problem in this setting. Here we also discuss possible adjustments in the modelling. The section 5 demonstrates an adapted resource allocation problem, where an additional state constraint is introduced. Besides of that this section is partly devoted to the formulation of a dual problem to some general infinite horizon control problem with state constraints and to the derivation of the sufficient optimality conditions based on the duality concept of Klötzler, see [10]. The obtained sufficient conditions allow us to prove that though the new setting of the problem admits an unbounded growth of the capital it possess an optimal solution having a "bang-singular" structure. Finally, brief conclusions are drawn.

\section{Preliminaries.}

Let us introduce $\mathbb{B}$ as a measurable set in $s$-dimensional Euclidean space. We denote by $\mathcal{M}^{n}(\mathbb{B}), L_{p}^{n}(\mathbb{B})$ and $C^{0, n}\left(\mathbb{R}^{+}\right)$the spaces of all vector functions $x: \mathbb{B} \rightarrow \mathbb{R}^{n}$ with Lebesgue measurable, in the $p$ th power Lebesgue integrable or continuous components, respectively ([5], p. 146 and pp. 285 ff.; [6], pp. 228 ff.). For $n=1$, we suppress the superscript in the labels of the spaces.

Let us write $[0, \infty)=\mathbb{R}^{+}$. A Lebesgue measurable function $\nu: \mathbb{R}^{+} \rightarrow$ $\mathbb{R}^{+} \backslash\{0\}$ with positive values is called a density function iff it is Lebesgue integrable over $\mathbb{R}^{+}$:

$$
L-\int_{0}^{\infty} \nu(t) d t<\infty
$$

By means of a density function $\nu \in C^{0}\left(\mathbb{R}^{+}\right)$, we define for any $1 \leq p<\infty$ the weighted Lebesgue space

$$
L_{p}^{n}(\mathbb{B}, \nu)=\left\{x \in \mathcal{M}^{n}(\mathbb{B}) \mid\left(L-\int_{\mathbb{B}}|x(t)|^{p} \nu(t) d t\right)^{1 / p}<\infty\right\}
$$

as well as 


$$
L_{\infty}^{n}(\mathbb{B}, \nu)=\left\{x \in \mathcal{M}^{n}(\mathbb{B})\left|\operatorname{ess} \sup _{\mathrm{t} \in \mathbb{B}}\right| \mathrm{x}(\mathrm{t}) \nu(\mathrm{t}) \mid<\infty\right\}
$$

and the weighted Sobolev space

$$
W_{p}^{1, n}\left(\mathbb{R}^{+}, \nu\right)=\left\{x \in \mathbb{M}^{n}\left(\mathbb{R}^{+}\right) \mid x \in L_{p}^{n}\left(\mathbb{R}^{+}, \nu\right), \dot{x} \in L_{p}^{n}\left(\mathbb{R}^{+}, \nu\right)\right\}
$$

(see [12], p. 11 f.). Equipped with the norm

$$
\|x\|_{W_{p}^{1, n}\left(\mathbb{R}^{+}, \nu\right)}=\|x\|_{L_{p}^{n}\left(\mathbb{R}^{+}, \nu\right)}+\|\dot{x}\|_{L_{p}^{n}\left(\mathbb{R}^{+}, \nu\right)},
$$

$W_{p}^{1, n}\left(\mathbb{R}^{+}, \nu\right)$ becomes a Banach space (this can be confirmed analogously to [12], p. 19, Theorem 3.6.).

The notation "L-" before an integral here and throughout the paper emphasizes that the improper integral has to be understood as Lebesgue integral. Let us remark that the corresponding Riemann integral does not necessarily coincide with the Lebesgue integral, see for example [6], [16].

\section{A Resource Allocation Problem with Finite Horizon}

\subsection{The problem $\left(P_{T}\right)$ and its solution by the Maximum Principle.}

We consider the resource allocation problem with a fixed finite horizon $T<\infty$, which is stated as follows

$$
J_{T}(x, u)=\int_{0}^{T} e^{-\rho t} x(t)(1-u(t)) \rightarrow \max !
$$

with respect to all pairs

$$
(x, u) \in W_{2}^{1, n}\left(\mathbb{R}^{+}\right) \times L_{2}^{r}\left(\mathbb{R}^{+}\right),
$$

satisfying

$$
\begin{aligned}
& \dot{x}(t)=x(t) \cdot u(t), \\
& x(0)=x_{0}>0, \\
& 0 \leq u(t) \leq 1
\end{aligned}
$$

In this setting the change rate of the capital stock $x(t)$ is proportional to the investment rate $u(t)$ at time $t$ which is to consider as the control variable. 
We now evaluate the necessary conditions given by Pontryagin's maximum principle on the interval $[0, T]$ and then decide if the candidate for an optimal solution computed by this way can be extended to a solution of the infinite horizon optimal control problem (6)-(10). In the normal case Pontryagin function is defined as follows

$$
\begin{aligned}
H(t, \xi, v, \eta) & =\xi(1-v) e^{-\rho t}+\eta \xi v \\
& =\xi e^{-\rho t}+v \xi\left(\eta-e^{-\rho t}\right)
\end{aligned}
$$

Maximizing Pontryagin function with respect to the variable $v$ we obtain the Hamiltonian

$$
\mathcal{H}(t, \xi, \eta)=\max _{v \in U} H(t, \xi, v, \eta)=\left\{\begin{aligned}
\eta \xi, \xi\left(\eta-e^{-\rho t}\right) & >0 \\
\xi e^{-\rho t}, \xi\left(\eta-e^{-\rho t}\right) & =0 \\
\xi^{-\rho t}, \xi\left(\eta-e^{-\rho t}\right) & <0
\end{aligned}\right.
$$

with the corresponding optimal control $u^{*}(t)$ :

$$
u^{*}(t)=\left\{\begin{aligned}
1, \xi\left(\eta-e^{-\rho t}\right) & >0 \\
\alpha \in[0,1], \xi\left(\eta-e^{-\rho t}\right) & =0 \\
0, \xi\left(\eta-e^{-\rho t}\right) & <0
\end{aligned}\right.
$$

where $\alpha$ is an arbitrary element of $[0,1]$. Due to concavity of $\mathcal{H}(t, \cdot, y(t))$ for all $t \in[0, T]$ the necessary conditions provided by the Maximum principle are sufficient as well, compare e.g. [7], theorem 2.2., p. 36. Therefore verifying conditions of the Pontryagin's maximum principle, theorem 1, [9], p. $127 \mathrm{ff}$., we find out that the process $\left(x^{*}, u^{*}, y\right)$, defined as

$$
\begin{aligned}
& u^{*}(t)=\left\{\begin{array}{l}
1, t \in(0, \tau), \\
0, t \in[\tau, T] ;
\end{array}\right. \\
& x^{*}(t)=\left\{\begin{array}{l}
x_{0} e^{t}, t \in(0, \tau), \\
x_{0} e^{\tau}, t \in[\tau, T] ;
\end{array}, t \in(0, \tau),\right. \\
& y(t)=\left\{\begin{array}{l}
D e^{-t} \\
\frac{1}{\rho}\left(e^{-\rho t}-e^{-\rho T}\right), t \in[\tau, T],
\end{array}\right.
\end{aligned}
$$

where $D=e^{(1-\rho) \tau}$ due to the switching condition $x^{*}(t)\left(y(t)-e^{-\rho t}\right)=0$, is optimal for a sufficiently large $T$. The point $\tau$ should be chosen such that the 
function $y$ remains continuous in this point. This gives us the condition

$$
e^{(1-\rho) \tau} e^{-\tau}=\frac{1}{\rho}\left(e^{-\rho \tau}-e^{-\rho T}\right)
$$

which yields the switching point

$$
\tau=T+\frac{\ln (1-\rho)}{\rho}>0
$$

Thus, if the interval $[0, T]$ is large enough then there exists a switching point $\tau$.

\section{The Resource Allocation Model on an Unbounded Time Interval.}

The extension of the problem $\left(\mathbf{P}_{\mathbf{T}}\right)$ onto an infinite horizon provides the optimal control problem

$$
J_{\infty}(x, u)=L-\int_{0}^{\infty} e^{-\rho t} x(t)(1-u(t)) d t \rightarrow \max !
$$

with respect to all pairs

$$
(x, u) \in W_{2}^{1,1}\left(\mathbb{R}^{+}\right) \times L_{2}^{1}\left(\mathbb{R}^{+}\right)
$$

fulfilling the restrictions

$$
\begin{aligned}
& \dot{x}(t)=x(t) \cdot u(t), \\
& x(0)=x_{0}>0, \\
& 0 \leq u(t) \leq 1
\end{aligned}
$$

where the discount rate $\rho$ satisfies $0<\rho<1$. The problem (19)-(23) will be denoted as $\left(\mathbf{P}_{\infty}\right)$.

\subsection{The problem $\left(\mathbf{P}_{\infty}\right)$ : Discussion.}

We now want to investigate what happens if we let the horizon $T$ tend to infinity. 
The first question which arises is about a suitable state space. Since for any admissible trajectory $x(t)$ it holds $x(t)>x_{0}>0$ for all $t \in[0, \infty)$ we observe that $x \notin W_{p}^{1,1}\left(\mathbb{R}^{+}\right)$for any $p \neq \infty$. This means that the feasible set of the problem $(\mathbf{P})_{\infty}$ is empty. However, considering the density function $\nu(t)=e^{-\rho t}$ we extend the state space $W_{2}^{1,1}\left(\mathbb{R}^{+}\right)$to $W_{2}^{1,1}\left(\mathbb{R}^{+}, e^{-\rho t}\right)$ and the control space $L_{2}^{1}\left(\mathbb{R}^{+}\right)$to $L_{2}^{1}\left(\mathbb{R}^{+}, e^{-\rho t}\right)$.

We now want to discuss the question of the existence of optimal solution for the infinite horizon control problem (19), (21)-(23) together with the condition

$$
(x, u) \in W_{2}^{1,1}\left(\mathbb{R}^{+}, e^{-\rho t}\right) \times L_{2}^{1}\left(\mathbb{R}^{+}, e^{-\rho t}\right) .
$$

For this aim we consider

$$
u_{\alpha}(t)=\alpha=\text { const } \in(0, \rho), \quad t \in \mathbb{R}^{+} .
$$

The corresponding state trajectory is

$$
x_{\alpha}(t)=x_{0} e^{\alpha t}, \quad t \in \mathbb{R}^{+},
$$

and the functional value given at the pair $\left(x_{\alpha}, u_{\alpha}\right)$ is

$$
\begin{aligned}
J_{\infty}\left(x_{\alpha}, u_{\alpha}\right) & =L-\int_{0}^{\infty} x_{0} e^{\alpha t}(1-\alpha) e^{-\rho t} d t \\
& =\left.\lim _{T \rightarrow \infty} \frac{(1-\alpha) x_{0}}{\alpha-\rho} e^{(\alpha-\rho) t}\right|_{0} ^{T}=\frac{1-\alpha}{\rho-\alpha} .
\end{aligned}
$$

It is easy to see that the sequence of functional values $J_{\infty}\left(x_{\alpha}, u_{\alpha}\right)$ tends to infinity as $\alpha \rightarrow \rho$. This fact indicates that there is no maximizer for the problem (19), (21)-(24) as well.

The last point of discussion is devoted to the question: how can we improve the given model so that the new one possess an optimal solution. Possible adjustments in the modelling are:

- Introducing an additional state constraint restricting the growth of the capital by some weight function. The obtained model makes sense in the economic context as well.

- Considering a nonlinear utility function such as suggested in [11], p. $238 \mathrm{ff}$.

- Is the state space to large? A more restrictive choice of the state space can resolve this problem.

- Logistic correction in the state equation

$$
\dot{x}(t)=x(t) u(t)-\beta x^{2}(t) .
$$


- Making the discount rate $\rho$ dependent on $t$.

\section{An Adapted Resource Allocation Model.}

We trace the first mentioned adjustment and modify the problem $\left(\mathbf{P}_{\infty}\right)$ by adding a state constraint and choosing weighted Sobolev- and weighted Lebesgue spaces as the state- and control spaces respectively:

$$
L-\int_{0}^{\infty} e^{-\rho t} x(t)(u(t)-1) d t \rightarrow \min !
$$

with respect to all pairs

$$
(x, u) \in W_{2}^{1}\left(\mathbb{R}^{+}, e^{-\alpha^{*} t}\right) \times L_{2}\left(\mathbb{R}^{+}, e^{-\alpha^{*} t}\right)
$$

satisfying the conditions

$$
\begin{aligned}
& \dot{x}(t)=x(t) \cdot u(t), \\
& x(t) \leq C e^{\hat{\alpha} t}, \quad \forall t>0, \quad C \geq x_{0}, \quad 0<\hat{\alpha}<\alpha^{*}<\rho, \\
& x(0)=x_{0}>0, \\
& 0 \leq u(t) \leq 1 .
\end{aligned}
$$

The problem (29)-(34) will be denoted by $\left(\mathbf{P}_{\infty}^{\mathbf{A}}\right)$.

\subsection{Sufficient optimality conditions through duality concept of Klötzler.}

The control problem which is the generalization of the $\left(\mathbf{P}_{\infty}^{\mathbf{A}}\right)$ can be formulated as follows: minimize the functional

$$
J_{\infty}^{G}(x, u)=L-\int_{0}^{\infty} r(t, x(t), u(t)) \tilde{\nu}(t) d t
$$

with respect to all pairs

$$
(x, u) \in W_{2}^{1, n}\left(\mathbb{R}^{+}, \nu\right) \times L_{2}^{r}\left(\mathbb{R}^{+}, \nu\right),
$$

satisfying 


$$
\begin{aligned}
& \dot{x}(t)=f(t, x(t), u(t)) \quad \text { a.e. on } \mathbb{R}^{+} ; \\
& x(t) \in Z(t) \text { on } \mathbb{R}^{+}, \\
& x(0)=x_{0} ; \\
& u(t) \in U \subset \operatorname{Compact}\left(\mathbb{R}^{\mathrm{r}}\right) \quad \text { a. e. on } \mathbb{R}^{+}
\end{aligned}
$$

Hereby the functions $\nu$ and $\tilde{\nu}$ are density functions described in the section 2 . The problem (35)-(40) will be denoted by $\left(\mathbf{P}_{\infty}^{\mathbf{G}}\right)$.

\section{Definition 1:}

We call a pair $(x, u)$ admissible for $\left(\mathbf{P}_{\infty}^{\mathbf{G}}\right)$, if it satisfies the conditions (36)-(40) and the integral in (35) exists and has a finite value.

\section{Definition 2:}

The pair $\left(x^{*}, u^{*}\right)$ is called optimal for $\left(\mathbf{P}_{\infty}^{\mathbf{G}}\right)$, if for any pair $(x, u)$ admissible for $\left(\mathbf{P}_{\infty}^{\mathbf{G}}\right)$ the inequality $J_{\infty}^{G}\left(x^{*}, u^{*}\right) \leq J_{\infty}^{G}(x, u)$ holds.

\section{Lemma 1:}

Let $\left(x^{*}, u^{*}\right)$ be an admissible pair of $\left(\mathbf{P}_{\infty}^{\mathbf{G}}\right)$ and $S: \mathbb{R}^{+} \times \mathbb{R}^{n} \rightarrow \mathbb{R}$ be a function of the form

$$
S(t, \xi)=a(t)+y(t)^{T}\left(\xi-x^{*}(t)\right)
$$

with $a \in W_{1}^{1}\left(\mathbb{R}^{+}\right) ; y \in W_{2}^{1}\left(\mathbb{R}^{+}, \nu^{-1}\right)$ and satisfying $y \in C^{1}[0, \tau], y \in C^{1}[\tau, \infty)$. Then, for any $x \in W_{2}^{1}\left(\mathbb{R}^{+}, \nu\right)$ with $x(0)=x_{0}$, we have:

$$
\begin{gathered}
\lim _{T \rightarrow \infty} S(T, x(T))=0 \\
\int_{0}^{\infty} \frac{d}{d t} S(t, x(t)) d t=-S\left(0, x_{0}\right)+S(\tau-0, x(\tau))-S(\tau+0, x(\tau)) .
\end{gathered}
$$

Proof: The proof is completely analogous to that of Lemma 1 in [15].

We introduce the Hamiltonian

$$
\mathcal{H}(t, \xi, \eta)=\sup _{v \in U} H(t, \xi, v, \eta)
$$

using the Pontryagin function

$$
H(t, \xi, v, \eta)=-r(t, \xi, v)+\frac{1}{\tilde{\nu}(t)}\langle\eta, f(t, \xi, v)\rangle
$$

Furthermore, we define the set 


$$
Y=\left\{\begin{array}{l|c}
S: \mathbb{R}^{+} \times \mathbb{R}^{n} \rightarrow \mathbb{R} & \begin{array}{c}
S(t, \xi)=a(t)+y(t)^{T}\left(\xi-x^{*}(t)\right), \\
a \in W_{1}^{1}\left(\mathbb{R}^{+}\right), y \in W_{2}^{1, n}\left(\mathbb{R}^{+}, \nu^{-1}\right), \\
y \in C^{1}[0, \tau], y \in C^{1}[\tau, \infty), \\
\frac{1}{\tilde{\nu}(t)} \partial_{t} S(t, \xi)+\mathcal{H}\left(t, \xi, \partial_{\xi} S(t, \xi)\right) \leq 0 \\
\forall(t, \xi) \in \mathbb{X},
\end{array}
\end{array}\right\}
$$

where

$$
\mathbb{X}=\left\{(t, \xi) \in \mathbb{R}^{+} \times \mathbb{R}^{n} \mid t \in\left(\mathbb{R}^{+} \backslash \tau\right), \xi \in Z(t)\right\}
$$

Using the construction scheme for the dual problem described in [10] we obtain a problem $\left(\mathbf{D}_{\infty}^{\mathbf{G}}\right)$ and prove

\section{Theorem 1:}

Let a problem $\left(\mathbf{P}_{\infty}^{\mathbf{G}}\right)$ be given. Then for the problem $\left(\mathbf{D}_{\infty}^{\mathbf{G}}\right)$ of maximizing the function

$$
\begin{gathered}
g_{\infty}(S):=-S\left(0, x_{0}\right)+\inf _{\beta \in Q}\{S(\tau-0, \beta)-S(\tau+0, \beta)\} \\
Q=\left\{\beta \in \mathbb{R}^{n} \mid \beta \in Z(\tau)\right\}
\end{gathered}
$$

with respect to $S \in Y$, the weak duality relation

$$
\inf \left(\mathbf{P}_{\infty}^{\mathbf{G}}\right) \geq \sup \left(\mathbf{D}_{\infty}^{\mathbf{G}}\right)
$$

holds.

\section{Proof:}

Let $(x, u)$ be admissible for $\left(\mathbf{P}_{\infty}^{\mathbf{G}}\right)$ and $S$ be admissible for $\left(\mathbf{D}_{\infty}^{\mathbf{G}}\right)$, i.e. $S \in Y$. Then we have

$$
\begin{aligned}
J(x, u) & =\int_{0}^{\infty} r(t, x(t), u(t)) \tilde{\nu}(t) d t \\
& =\int_{0}^{\infty}\left(-H\left(t, x(t), u(t), \partial_{\xi} S(t, x(t))\right)\right) \tilde{\nu}(t) d t \\
& +\int_{0}^{\infty}\left(\frac{\partial_{\xi} S(t, x(t))}{\tilde{\nu}(t)} f(t, x(t), u(t))\right) \tilde{\nu}(t) d t \\
& =\int_{0}^{\infty}\left(-H\left(t, x(t), u(t), \partial_{\xi} S(t, x(t))\right)-\frac{\partial_{t} S(t, x(t))}{\tilde{\nu}(t)}\right) \tilde{\nu}(t) d t
\end{aligned}
$$




$$
\begin{aligned}
& +\int_{0}^{\infty}\left(\frac{\partial_{t} S(t, x(t))}{\tilde{\nu}(t)}+\frac{\partial_{\xi} S(t, x(t))}{\tilde{\nu}(t)} \dot{x}(t)\right) \tilde{\nu}(t) d t \\
& \geq-\int_{0}^{\infty}\left(\mathcal{H}\left(t, x(t), \partial_{\xi} S(t, x(t))+\frac{\partial_{t} S(t, x(t))}{\tilde{\nu}(t)}\right) \tilde{\nu}(t) d t\right. \\
& +\int_{0}^{\infty}\left(\partial_{t} S(t, x(t))+\partial_{\xi} S(t, x(t)) \dot{x}(t)\right) d t \\
& \geq-\int_{0}^{\infty} \sup _{\xi \in \mathbb{R}^{n}}\left\{\left(\mathcal{H}\left(t, \xi, \partial_{\xi} S(t, \xi)\right)+\frac{\partial_{t} S(t, \xi)}{\tilde{\nu}(t)}\right)\right\} \tilde{\nu}(t) d t \\
& +\int_{0}^{\infty}\left(\partial_{t} S(t, x(t))+\partial_{\xi} S(t, x(t)) \dot{x}(t)\right) d t \\
& \geq \int_{0}^{\infty} \frac{d}{d t} S(t, x(t)) d t=\lim _{T \rightarrow \infty} \int_{0}^{T} \frac{d}{d t} S(t, x(t)) d t \\
& =\lim _{T \rightarrow \infty} S(T, x(T))-S(0, x(0))+S(\tau-0, x(\tau))-S(\tau+0, x(\tau)) \\
& \geq-S\left(0, x_{0}\right)+\inf _{\beta \in Q}\{S(\tau-0, \beta)-S(\tau+0, \beta)\} .
\end{aligned}
$$

The next corollary provides sufficient conditions for the global optimality.

\section{Corollary 1:}

An admissible pair $\left(x^{*}, u^{*}\right)$ is a global minimizer of $\left(\mathbf{P}_{\infty}^{\mathbf{G}}\right)$, if there exists an admissible $S^{*}$ for $\left(\mathbf{D}_{\infty}^{\mathbf{G}}\right)$, such that the following conditions are fulfilled for almost all $t>0$ :

$(\mathrm{M})$

$$
\begin{aligned}
(\mathbf{M}) \quad \mathcal{H}\left(t, x^{*}(t), \partial_{\xi} S^{*}\left(t, x^{*}(t)\right), 1\right) & =H\left(t, x^{*}(t), u^{*}(t), \partial_{\xi} S^{*}\left(t, x^{*}(t)\right), 1\right), \\
(\mathbf{H J E}) \quad & \frac{1}{\tilde{\nu}(t)} \partial_{t} S^{*}(t, \\
& \left.x^{*}(t)\right)+\mathcal{H}\left(t, x^{*}(t), \partial_{\xi} S\left(t, x^{*}(t)\right)\right)=0, \\
(\mathbf{B}) \quad \inf _{\beta \in Q}\left\{S^{*}(\tau-\right. & \left.0, \beta)-S^{*}(\tau+0, \beta)\right\} \\
& =S^{*}\left(\tau-0, x^{*}(\tau)\right)-S^{*}\left(\tau+0, x^{*}(\tau)\right) .
\end{aligned}
$$

Proof: This follows immediately from Theorem 1 and Lemma 1.

\subsection{Optimal solution of $\left(\mathbf{P}_{\infty}^{\mathbf{A}}\right)$ by sufficiency conditions.}

We consider again the problem $\left(\mathbf{P}_{\infty}^{\mathbf{A}}\right)$ and show that the control 


$$
u^{*}(t)=\left\{\begin{array}{l}
1, t \leq \tau \\
\hat{\alpha}, t>\tau
\end{array}\right.
$$

is optimal for some switching point $\tau$. The corresponding state trajectory is

$$
x^{*}(t)=\left\{\begin{array}{c}
x_{0} e^{t} \quad, t \leq \tau, \\
x_{0} e^{(1-\hat{\alpha}) \tau} e^{\hat{\alpha} t}, t>\tau .
\end{array}\right.
$$

This solution satisfies the state constraint (32) if $x_{0} e^{\tau}=C e^{\hat{\alpha} \tau}$, which means that $\tau=\frac{1}{1-\hat{\alpha}} \ln \left(\frac{C}{x_{0}}\right) \geq 0$. We now want to find an $S^{*}$, admissible in $\left(\mathbf{D}_{\infty}^{\mathbf{A}}\right)$, such that the conditions (52), (53) and (54) are fulfilled for the pair $\left(x^{*}, u^{*}\right)$. This ensures the global optimality of the process $\left(x^{*}, u^{*}\right)$.

The Hamiltonian for the problem $\left(\mathbf{P}_{\infty}^{\mathbf{A}}\right)$ is defined by

$$
\mathcal{H}(t, \xi, \eta)=\max _{v \in U} H(t, \xi, v, \eta)=\left\{\begin{aligned}
\eta \xi e^{\rho t}, \xi\left(\eta e^{\rho t}-1\right) & >0 \\
\xi, \xi\left(\eta e^{\rho t}-1\right) & =0 \\
\xi, \xi\left(\eta e^{\rho t}-1\right) & <0
\end{aligned}\right.
$$

We consider the parametric optimization problem of maximizing the defect within the Hamilton-Jacobi equation (53):

$$
\begin{array}{r}
\Lambda(t, \xi):=\frac{1}{\tilde{\nu}(t)} \partial_{t} S(t, \xi)+\mathcal{H}\left(t, \xi, \partial_{\xi} S(t, \xi)\right) \rightarrow \max ! \\
\text { with respect to } \xi \in\left\{\xi \in \mathbb{R} \mid x_{0} \leq \xi \leq C e^{\hat{\alpha} t}\right\} .
\end{array}
$$

The defect $\Lambda(t, \xi)$ from the last formula can be rewritten as

$$
\begin{aligned}
\Lambda(t, \xi) & =\frac{1}{\tilde{\nu}(t)}\left(\dot{a}(t)+\dot{y}(t)^{T}\left(\xi-x^{*}(t)\right)-y(t)^{T} \dot{x}^{*}(t)\right) \\
& +\mathcal{H}\left(t, \xi, \partial_{\xi} S(t, \xi)\right) .
\end{aligned}
$$

The component $a(t)$ of the dual variable $S(t, \xi)$ will be chosen from the Hamilton-Jacobi equation $\Lambda\left(t, x^{*}(t)\right)=0$ and we obtain

$$
\dot{a}(t)=\left(y(t)^{T} \dot{x}^{*}(t)-\mathcal{H}\left(t, x^{*}(t), y(t)\right)\right) \tilde{\nu}(t) .
$$

With the Lagrangian

$$
L(t, \xi)=-\Lambda(t, \xi)+\lambda(t)\left(x_{0}-\xi\right)+\mu(t)\left(\xi-C e^{\alpha t}\right)
$$


the Karush-Kuhn-Tucker conditions for the problem (58) are

$$
\begin{aligned}
\partial_{\xi} L\left(t, x^{*}(t)\right)= & -e^{\rho t} \dot{y}(t)-\partial_{\xi} \mathcal{H}\left(t, x^{*}(t), y(t), 1\right)-\lambda(t)+\mu(t)=0, \\
& \lambda(t)\left(x_{0}-x^{*}(t)\right)=0, \quad \lambda(t) \geq 0, \\
& \mu(t)\left(x^{*}(t)-C e^{\alpha t}\right)=0, \quad \mu(t) \geq 0 .
\end{aligned}
$$

Since $u^{*}(t)=1$ for all $t \in(0, \tau)$, the strict inequalities $x_{0}<x^{*}(t)<C e^{\hat{\alpha} t}$ hold for all $t$ in this interval, which means together with complementary conditions (63), (64) that $\lambda(t)=\mu(t)=0$ on $(0, \tau)$. In this case the condition (62) turns into

$$
\dot{y}(t)=-y(t), \quad t \in(0, \tau)
$$

and, consequently, $y(t)=D e^{-t}, D \in \mathbb{R}$. From the condition $D e^{-t}>e^{-\rho t} \forall t \in$ $(0, \tau)$ we choose the constant $D:=e^{(1-\rho) \tau}$.

The fact that $u^{*}(t)=\hat{\alpha} \in(0,1)$ for all $t \in(\tau, \infty)$ implies $\mathcal{H}(t, \xi, \eta)=\xi$ and $\lambda(t)=0, t \in(\tau, \infty)$. For all $t \in(\tau, \infty)$ we rewrite the condition (62) into the form

$$
\dot{y}(t)=-e^{-\rho t}+\mu(t) e^{-\rho t} .
$$

Together with the switching condition $y(t)=e^{-\rho t}$ for all $(\tau, \infty)$ we obtain the equation with respect to the multiplier $\mu(t)$ :

$$
-\rho e^{-\rho t}=-e^{-\rho t}+\mu(t) e^{-\rho t}
$$

whose solution $\mu(t)=(1-\rho) e^{-\rho t}$ is strictly positive.

The continuity of the adjoint function $y(t)$ at the point $\tau$ indicates that the boundary condition (54) of the generalized maximum principle is satisfied by the triple $\left(x^{*}(t), u^{*}(t), S^{*}\left(t, x^{*}(t)\right)\right)$, since in this case the both sides of $(54)$ are zero.

Finally, we prove that the adjoint function $y(t)$ belongs to the space $W_{2}^{1}\left(\mathbb{R}^{+}, e^{\alpha^{*} t}\right)$ :

$$
\begin{aligned}
\|y\|_{L_{2}\left(\mathbb{R}^{+}, e^{\alpha^{*}}\right)} & =\int_{0}^{\infty}|y(t)|^{2} e^{\alpha^{*} t} d t \\
& =e^{2(1-\rho) \tau} \int_{0}^{\tau} e^{-\left(2-\alpha^{*}\right) t} d t+\int_{\tau}^{\infty} e^{-\left(2 \rho-\alpha^{*}\right) t} d t<\infty .
\end{aligned}
$$

We analogously show that $\|\dot{y}\|_{L_{2}\left(\mathbb{R}^{+}, e^{\alpha^{*} t}\right)}<\infty$ holds. It remains to verify, whether $a(t)$ from $(60)$ belongs to the space $W_{1}^{1}\left(\mathbb{R}^{+}\right)$. Indeed, the function $a(t)$, given by 


$$
a(t)=\left\{\begin{array}{c}
x_{0} \frac{\hat{\alpha}-1}{\hat{\alpha}-\rho} e^{(1-\rho) \tau}+K \quad, t<\tau, \\
x_{0} \frac{\hat{\alpha}-1}{\hat{\alpha}-\rho} e^{(1-\hat{\alpha}) \tau} e^{(\hat{\alpha}-\rho) t}+K, t>\tau,
\end{array}\right.
$$

where $K \in \mathbb{R}$, is obviously an element of the $W_{1}^{1}\left(\mathbb{R}^{+}\right)$.

Thus, all the conditions of the corollary 1 are fulfilled and we conclude that the pair $\left(x^{*}, u^{*}\right)$ is the global minimizer of the problem $\left(\mathbf{P}_{\infty}^{\mathbf{A}}\right)$ and the proof is complete.

\section{Conclusions.}

To summarize, we want to remark that using the weight functions approach we have succeeded in changing the model statement in so far that the problem $\left(\mathbf{P}_{\infty}^{\mathbf{A}}\right)$ became solvable. Further, the duality concept of Klötzler allowed us to formulate the sufficient optimality conditions and to prove the global optimality of the control (55). However, an equivalent of Pontryagin's maximum principle for the problem $\left(\mathbf{P}_{\infty}^{\mathbf{G}}\right)$ with adjoint variables belonging to dual spaces of the state spaces, including correct transversality conditions is still missing and represents a future challenge.

\section{References}

[1] Benveniste, L.M., Scheinkman, J.A. (1982). Duality Theory for Dynamic Optimization Models of Economics: The Continuous Time Case. Journal of Economic Theory 27, 1-19.

[2] Blot, J.; Cartigny, P. (1995). Bounded Solutions and Oscillations of Concave Lagrangian Systems in Presence of a Discount Rate. Journal for Analysis and its Applications14, pp.731-750.

[3] Carlson, D.A., A.B. Haurie and A. Leizarowitz (1991). Infinite Horizon Optimal Control, Springer-Verlag, New York, Berlin, Heidelberg.

[4] Dmitruk A.V., Kuz'kina N.V. (2005). Existence theorem in the Optimal Control Problem on an Infinite Time Interval, Mathematical Notes 78, No.4, 466-480.

[5] Dunford N.; Schwartz, J. T. (1988). Linear Operators. Part I: General Theory. Wiley-Interscience; New York, etc.

[6] Elstrodt, J. (1996). Maß und Integrationstheorie, Springer, Berlin.

[7] Feichtinger, G.; Hartl, R. F. (1986). Optimale Kontrolle ökonomischer Prozesse. de Gruyter; Berlin - New York. 
[8] Halkin, H. (1979). Necessary conditions for optimal control problems with infinite horizons. Econometrica 42, $267-272$.

[9] Ioffe, A. D.; Tichomirow, V. M. (1979). Theorie der Extremalaufgaben, VEB Deutscher Verlag der Wissenschaften, Berlin.

[10] Klötzler, R. (1979): On a general conception of duality in optimal control. In: Equadiff IV. Proceedings of the Czechoslovak Conference on Differential Equations and their Applications held in Prague, August 22 - 26, 1977. (Fábera, J. (Ed.)), (Lecture Notes in Mathematics 703), 189 - 196, Springer, Berlin.

[11] Korn, R.; Korn, E. (1999). Optionsbewertung und Portfoliooptimierung, GABLER Vieweg, Braunschweig/Wiesbaden.

[12] Kufner, A. (1985). Weighted Sobolev Spaces. John Wiley \& Sons; Chichester, etc.

[13] Leizarowitz, A.; Mizel, V. J. (1989). One-dimensional infinite-horizon variational problems arising in continuum mechanics. Arch. Rat. Mech. Anal. 106, 161 - 194.

[14] Magill, M. J. P.(1982). Pricing infinite horizon programs. J. Math. Anal. Appl. $\mathbf{8 8}, 398-421$.

[15] Pickenhain, S.; Lykina, V. (2006). Sufficiency conditions for infinite horizon optimal control problems. In: Recent Advances in Optimization. (Seeger, A. (Ed.)), (Lecture Notes in Economics and Mathematical Systems 563), 217 232, Springer, Berlin, etc.

[16] Pickenhain, S.; Lykina, V.; Wagner, M. (2006). Lebesgue and improper Riemann integrals in infinite horizon optimal control problems. Preprint Nr. M-02/2006, Reihe Mathematik, BTU Cottbus, Cottbus.

[17] Sethi, S. P.; Thompson, G. L. (2000). Optimal Control Theory. Applications to Management Science and Economics. Kluwer; Boston - Dordrecht - London, 2nd ed.

[18] Zaslavski, A. J. (1995). The existence of periodic minimal energy configurations for one-dimensional infinite horizon variational problems arising in continuum mechanics. J. Math. Anal. Appl. 194, $459-476$. 